\title{
Linear and nonlinear decoupling using time-dependent transformations
}

\author{
Andrzej Wolski* \\ Department of Physics, The University of Liverpool, Liverpool, L69 7ZE, United Kingdom \\ and The Cockcroft Institute, Daresbury, Warrington, WA4 4AD, United Kingdom \\ Andrew M. Sessler \\ Lawrence Berkeley National Laboratory, Berkeley, California 94720, USA
}

(Received 2 August 2006; published 13 February 2007)

\begin{abstract}
Linear coupling in a storage ring is conveniently analyzed in terms of transformations that put the single-turn map into block-diagonal form. Such a transformation allows us to define new variables, in which the dynamics are uncoupled. In this paper it is shown how a similar approach may be taken to nonlinear coupling, but that to decouple the map completely one needs to use a time-dependent canonical transformation. In Sec. III, we present a numerical example, based upon the analysis presented in previous sections, of a nonlinear transformation. In part for pedagogical reasons, and in part to make our use of notation clear, in Appendix A we reproduce the theory, along with a numerical example, of the wellknown result for a linear transformation.
\end{abstract}

DOI: 10.1103/PhysRevSTAB.10.024002

PACS numbers: 29.20.Dh, 29.27.Bd

\section{INTRODUCTION}

Making transformations to reduce a dynamical system to as simple a form as possible is a familiar procedure in accelerator physics. For example, in the case of linear, uncoupled motion in a periodic lattice, a canonical transformation to variables in which the phase space portrait is a circle yields the Twiss parameters. In the case of a nonlinear system, normal form analysis (in which nonlinear terms of progressively higher order are removed by appropriate canonical transformations) yields useful information on the nonlinearities, from the form of the transformations required.

A system in which there is coupling between dynamical degrees of freedom is one example where a transformation to variables that simplify the motion can be a useful procedure. Coupling is an ubiquitous phenomenon in accelerator beam lines, arising from many sources, including the tilt of normal quadrupoles around the beam axis and vertical offsets of the beam in sextupoles. The presence of coupling complicates the description of the dynamics. For example, consider the phase space picture produced by a particle making multiple turns through a linear storage ring lattice. If the motion is symplectic and uncoupled, the values of the dynamical variables of the particle in any degree of freedom trace out (over time) smooth ellipses in phase space, with the area of each ellipse corresponding to a conserved quantity of the motion. Coupling can affect the motion in such a way that a smooth boundary enclosing a well-defined area in phase space can no longer be clearly identified, although usually there are still conserved quantities. By finding a canonical decoupling transformation,

*Electronic address: a.wolski@dl.ac.uk we can construct variables in which the motion in each degree of freedom does lie on a smooth ellipse, with the area of each ellipse corresponding to a conserved quantity.

In the case of linear coupling, it is well known how to find a canonical transformation to new coordinates in which the motion is uncoupled. In Appendix A, we review the general theory of linearly coupled motion together with a numerical example. Partly, this serves to introduce our notation (Lie algebraic methods), but the analysis is also pedagogically useful. Also well known is the normal form procedure for removing terms from nonlinear maps that drive resonances, including coupling resonances, and hence distort the phase space ellipses observed with uncoupled linear motion. However, when performing a normal form analysis of a coupled map, usually there remain coupling terms that give a dependence on the phase advance in one plane to the amplitude in another plane. How to remove these remaining coupling terms has not been described in the literature, and is the subject of this paper. Briefly, we find that, to decouple completely a coupled system, a time-dependent canonical transformation is needed. The analysis is presented in the body of the paper and a (rather extensive) numerical example is given in Sec. III.

One interesting aspect of the complete decoupling is that it provides an explicit and simple geometrical manifestation of Liouville's theorem. Consider the case of a coupled system, in which the areas of the phase space ellipses in each degree of freedom correspond to conserved quantities, but where the coupling leads to the phase advance in one degree of freedom having a dependence on the amplitude of the motion in another degree of freedom. In this case, particles covering a given volume in phase space can spread to cover an apparently larger (or smaller) area in one 
degree of freedom, depending on the values of their phase space coordinates in another degree of freedom. If the coupling is completely removed by canonical transformations, the phase space area covered by a set of particles remains constant in each degree of freedom. Liouville's theorem, which states that the density of points in phase space remains constant during the evolution of a symplectic system, is thus evident within each degree of freedom separately.

The approach presented here provides some insight into the nature of the dynamics in systems with nonlinear coupling. Although we have not yet used this technique either in the analysis of a real accelerator or in solving problems presented in the design of new systems, we are motivated to describe this approach for it does provide an extension to the conventional way of thinking about coupled nonlinear Hamiltonian systems. There are indications in the numerical example in Sec. III that application of time-dependent decoupling transformations can be used to obtain information on the limits on the amplitudes for which the dynamics of a coupled system are stable.

\section{NONLINEAR COUPLING}

Appendix A reviews decoupling of linear maps: the linear analysis presented there generalizes to the nonlinear case, which we now consider. For convenience, we work in action angle rather than Cartesian variables. In general, the decoupling proceeds in two steps. First, we normalize the map, in the sense that we eliminate any dependence on the angle variables. Any remaining coupling terms will then involve only the action variables. In the second step, we remove these terms; we will show that, in general, a timedependent canonical transformation is needed to do this.

\section{A. Normal-form analysis}

Techniques for constructing a transformation to remove dependencies of a nonlinear map on the angle variables are familiar from normal form theory [1]. As an example, consider the map (expressed as a Lie transform [2]):

$$
M=R \cdot \exp \left(: \bar{m}_{2}:\right)
$$

where $R=R\left(\mu_{x}, \mu_{y}\right)$ is a linear rotation through angles $\mu_{x}$ and $\mu_{y}$, and

$$
\bar{m}_{2}=\kappa J_{x} J_{y} \cos \left(2 \phi_{x}+2 \phi_{y}\right) .
$$

In general, the linear part of the map represented by $R$ may include coupling; however, the linear map may be decoupled using the techniques described in Appendix A. To address the nonlinear part, we apply a canonical transformation $F_{2}$ that is second order in the action variables:

$$
M \mapsto \tilde{M}=F_{2} \cdot M \cdot F_{2}^{-1},
$$

where

$$
\begin{aligned}
\tilde{M} & =R \cdot R^{-1} \cdot F_{2} \cdot R \cdot \exp \left(: \bar{m}_{2}:\right) \cdot F_{2}^{-1} \\
& =R \cdot \exp \left(: R^{-1} \bar{f}_{2}:\right) \cdot \exp \left(: \bar{m}_{2}:\right) \cdot \exp \left(:-\bar{f}_{2}:\right) .
\end{aligned}
$$

$\bar{f}_{2}$ is the generator of the transformation $F_{2}$ :

$$
F_{2}=\exp \left(: \bar{f}_{2}:\right) \text {. }
$$

The Baker-Campbell-Hausdorff $(\mathrm{BCH})$ formula gives

$$
\exp (: A:) \exp (: B:)=\exp \left(: A+B+\frac{1}{2}[A, B]+O:\right),
$$

where $A$ and $B$ are functions of the dynamical variables, $[A, B]$ is the Poisson bracket of $A$ and $B$, and $O$ represents terms involving higher-order products of $A$ and $B$. Applying the $\mathrm{BCH}$ formula (3) to Eq. (2) gives

$$
\tilde{M}=R \cdot \exp \left(: \bar{m}_{2}^{\prime}+\bar{m}_{3}^{\prime}+O(4):\right),
$$

where $\bar{m}_{2}^{\prime}$ is a second-order polynomial in $J_{x}$ and $J_{y}$ :

$$
\bar{m}_{2}^{\prime}=R^{-1} \bar{f}_{2}+\kappa J_{x} J_{y} \cos \left(2 \phi_{x}+2 \phi_{y}\right)-\bar{f}_{2} .
$$

$\bar{m}_{3}^{\prime}$ is a third-order polynomial in $J_{x}$ and $J_{y}$ arising from the Poisson bracket shown explicitly in Eq. (3), and $O(4)$ represents fourth- and higher-order polynomials in $J_{x}$ and $J_{y}$. To eliminate the second-order term (in other words, to set $\bar{m}_{2}^{\prime}=0$ ), the generator $\bar{f}_{2}$ must satisfy

$$
\left(1-R^{-1}\right) \bar{f}_{2}=\kappa J_{x} J_{y} \cos \left(2 \phi_{x}+2 \phi_{y}\right) .
$$

Since the variables $J_{x}$ and $J_{y}$ are invariant under a rotation, and

$$
R^{-1} \phi_{x(y)}=\phi_{x(y)}-\mu_{x(y)},
$$

we find that Eq. (4) has the solution

$$
\bar{f}_{2}=\frac{1}{2} J_{x} J_{y} \frac{\sin \left(\mu_{x}+\mu_{y}+2 \phi_{x}+2 \phi_{y}\right)}{\sin \left(\mu_{x}+\mu_{y}\right)} .
$$

In eliminating the unwanted second-order terms, we introduce additional third-order (and higher) terms. Any terms not invariant under rotations may be removed by applying the same procedure. Assuming convergence (which may not necessarily occur, particularly in regions where the motion appears chaotic), the normalization proceeds order by order until the desired accuracy is achieved.

The third-order term $\bar{m}_{3}^{\prime}$ remaining in the map (after eliminating the second-order terms with the transformation $F_{2}$ ) arises from the Poisson bracket in the $\mathrm{BCH}$ formula (3), and is given by

$$
\bar{m}_{3}^{\prime}=\frac{1}{4} \kappa^{2} J_{x} J_{y}\left(J_{x}+J_{y}\right) \frac{\sin \left[2\left(\mu_{x}+\mu_{y}\right)\right]}{\sin ^{2}\left(\mu_{x}+\mu_{y}\right)} .
$$

We see that, in this case, $\bar{m}_{3}^{\prime}$ is invariant under rotations and cannot be removed by the normal form procedure described above: we shall discuss how to deal with such terms in the following sections, and return to this particular example in Sec. III. However, at this point, it is possible to find the invariants of the nonlinear map. In the transformed 
variables, the invariants are simply $\tilde{J}_{x}$ and $\tilde{J}_{y}$, where, assuming that the normalization has proceeded up to order $n$,

$$
\tilde{J}_{x(y)}=\prod_{i=n}^{2} F_{i} \cdot J_{x(y)} .
$$

The invariants in the original variables are obtained by applying the inverse transformations to $\tilde{J}_{x}$ and $\tilde{J}_{y}$.

\section{B. Nonlinear decoupling of Hamiltonians}

Clearly, terms in the generator that are invariant under rotations cannot be removed by the above procedure: coupling terms such as $J_{x} J_{y}$ may remain. To find a means of eliminating these terms, let us consider the analogous situation in Hamiltonian dynamics; this has been treated by Schräpel [3]. We will take as the Hamiltonian

$$
H=\mu_{x} J_{x}+\mu_{y} J_{y}+\kappa J_{x} J_{y}
$$

The independent variable will be $t$, which does not appear explicitly in the Hamiltonian. We now write down a generating function of the second kind [4]:

$$
F_{2}=\phi_{x} \tilde{J}_{x}+\phi_{y} \tilde{J}_{y}-\kappa \tilde{J}_{x} \tilde{J}_{y} t,
$$

where $\tilde{J}_{x}$ and $\tilde{J}_{y}$ are the new action variables. Note the explicit appearance of $t$ in the generating function. The old action variables are given in terms of the new variables by

$$
J_{x(y)}=\frac{\partial F_{2}}{\partial \phi_{x(y)}}=\tilde{J}_{x(y)},
$$

and the new angle variables are given by

$$
\tilde{\phi}_{x(y)}=\frac{\partial F_{2}}{\partial \tilde{J}_{x(y)}}=\phi_{x(y)}-\kappa \tilde{J}_{y(x)} t=\phi_{x(y)}-\kappa J_{y(x)} t .
$$

The transformation takes us into a rotating frame of reference, in which the rate of rotation in either plane depends on the action in the other plane. The transformed Hamiltonian $\tilde{H}$ is given by

$$
\tilde{H}=H+\frac{\partial F_{2}}{\partial t}=\mu_{x} \tilde{J}_{x}+\mu_{y} \tilde{J}_{y} .
$$

In the new variables, the motion is uncoupled: we have achieved our goal by means of a generating function in which the independent variable $t$ appears explicitly.

\section{Nonlinear decoupling of maps}

We now return to the case of a map with nonlinear coupling. For maps, the equivalent of a time-dependent transformation that we saw for Hamiltonians is a transformation that depends on "turn" number, $n$ (i.e. the number of times that the map is applied to the variables). In effect, we need an infinite set of decoupling transformations, indexed by the turn number. We assume that we have decoupled the linear part, and normalized the nonlinear part so that it contains terms only in $J_{x}$ and $J_{y}$. As an explicit example, consider

$$
M=R \cdot \exp \left(: \kappa J_{x} J_{y}:\right)
$$

The nonlinear part cannot be decoupled using a canonical transformation that is independent of the turn number, $n$; however, let us consider a canonical transformation, $F(n)$, that has an explicit dependence on the turn number [or, put another way, $F(n)$ represents a set of transformations indexed by $n$ ]. Under the map $M$ and under the transformation $F(n)$, the variables transform as follows:

$$
\mathbf{J}_{n+1}=M \cdot \mathbf{J}_{n} \quad \tilde{\mathbf{J}}_{n}=F(n) \cdot \mathbf{J}_{n} .
$$

It follows that under $F(n), M$ transforms as

$$
M \mapsto \tilde{M}=F(n+1) \cdot M \cdot F^{-1}(n) .
$$

An appropriate form for the generating function is

$$
F(n)=\exp \left(:-\kappa J_{x, n} J_{y, n} n:\right),
$$

in which case the fully decoupled map is simply

$$
\tilde{M}=R \text {. }
$$

It appears that, by applying successive canonical transformations, we have "lost" a wealth of information contained in the original map, and reduced it to a mere linear rotation. However, by referring back to the linear case, we can understand what has happened: the full dynamics in the system are no longer contained in the map itself, but are now contained in the canonical transformations that we used to simplify the map to a point where the dynamics are trivial to describe. The nature of the various canonical transformations in themselves indicates something about the dynamical features in the original map; that we need a turn-dependent canonical transformation to decouple the map completely indicates the presence of coupling terms that are invariant under phase advances, i.e., under transformations of the angle variables $\phi_{x(y)} \mapsto \phi_{x(y)}+\mu_{x(y)}$

\section{NUMERICAL EXAMPLE}

Recall the example that we used for the normal form procedure in Sec. II A, Eq. (1):

$$
M=R \cdot \exp \left[: \kappa J_{x} J_{y} \cos \left(2 \phi_{x}+2 \phi_{y}\right):\right],
$$

where $R$ is a linear map with phase advances $\mu_{x}$ and $\mu_{y}$. After applying a second-order normalizing transformation (5), the transformed map is

$$
\tilde{M}=R \cdot \exp \left(: \bar{m}_{3}^{\prime}+O(4):\right),
$$

where the third-order generator term $\bar{m}_{3}^{\prime}$ is given by (6)

$$
\bar{m}_{3}^{\prime}=\frac{1}{4} \kappa^{2} J_{x} J_{y}\left(J_{x}+J_{y}\right) \frac{\sin \left[2\left(\mu_{x}+\mu_{y}\right)\right]}{\sin ^{2}\left(\mu_{x}+\mu_{y}\right)} .
$$

As we observed in Sec. II A, $\bar{m}_{3}^{\prime}$ is invariant under rota- 
tions, so cannot be removed using the normal form procedure with turn-independent transformations; however, from Sec. IIC we can construct a turn-dependent transformation, with generator

$$
\bar{f}_{3}(t)=-\bar{m}_{3}^{\prime} n,
$$

which will complete the decoupling to third order.

The results of tracking 18 turns (with $\kappa=0.85$, and linear rotation angles $\mu_{x}=100^{\circ}$ and $\mu_{y}=65^{\circ}$ ) are shown in Fig. 1. The blue and black points show the results of applying the map $M$ to particles with two different initial vertical amplitudes. The second-order turn-independent normalization reduces the scatter observed in the full nonlinear map; and the invariants of the motion now correspond to the areas of the ellipses. However, the motion is still coupled since the horizontal phase advance depends on the vertical amplitude (and vice versa). After a third-order
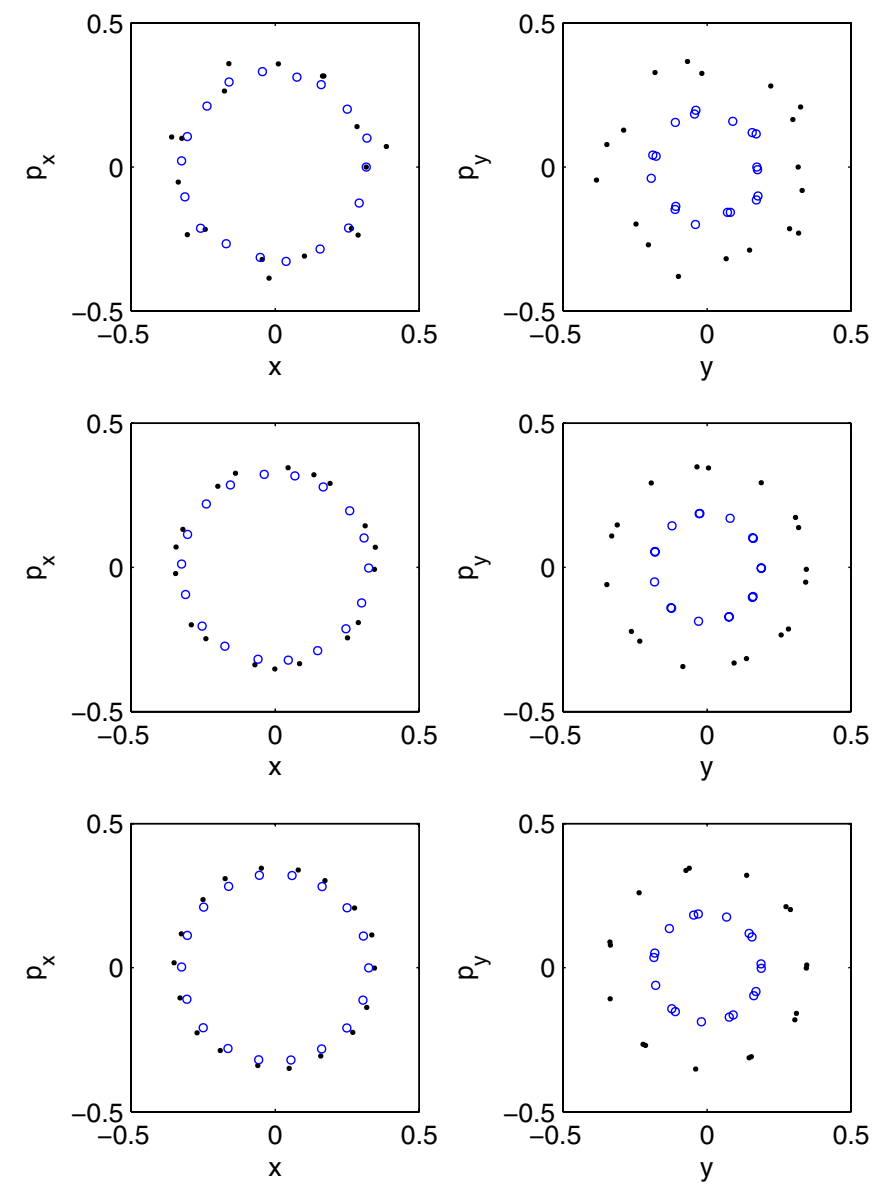

FIG. 1. (Color) Nonlinear decoupling. Top: nonlinear map. Middle: map normalized using second-order turn-independent canonical transformation. Bottom: map further normalized using third-order turn-dependent canonical transformation. The solid black points show the result of mapping with initial values for the actions $J_{x}=0.05, J_{y}=0.05$; the open blue circles show the results of mapping with initial values for the actions $J_{x}=0.05$, $J_{y}=0.015$. turn-dependent normalization, the phase advance in each plane is independent of the amplitude in the other plane: the motion is almost fully decoupled.

The effect of the final (turn-dependent) decoupling transformation can be seen most clearly by calculating the horizontal tune shifts as a function of vertical amplitude. Analytically, the tune shift with amplitude may be found directly from the normalizing transformation. First, observe that a linear rotation may be written simply as

$$
R\left(\mu_{x}, \mu_{y}\right)=\exp \left(:-\mu_{x} J_{x}-\mu_{y} J_{y}:\right) .
$$

Next, we recall that a turn-independent normalization removes only terms in the generator that have a dependence on the angle variables. The terms that remain give the tunes as functions of the action variables; specifically, the tunes are the coefficient of $J_{x}$ and $J_{y}$ after the turn-independent normalization. For our example Eq. (7), we see immediately from Eq. (6) that the horizontal tune shift can be written to second order in the action variables as

$$
\Delta \nu_{x} \approx-\frac{1}{8 \pi} \kappa^{2} J_{y}\left(J_{x}+J_{y}\right) \frac{\sin \left[2\left(\mu_{x}+\mu_{y}\right)\right]}{\sin ^{2}\left(\mu_{x}+\mu_{y}\right)} .
$$

Numerically, the tunes as functions of amplitude may be obtained by applying the map some number of times to a point with given initial values for the action variables; then applying a Fourier analysis to the resulting data. The precision of the tune obtained in this way can be improved, as we do here, by using an interpolated Fourier-Hanning technique, instead of a straightforward Fourier analysis.

Figure 2 shows a comparison between the analytical estimate, Eq. (8), of the horizontal tune shift with vertical amplitude and numerical results for the original map, Eq. (7). We use the same values for $\kappa, \mu_{x}$, and $\mu_{y}$ as before, and a fixed initial value of $10^{-4}$ for $J_{x}$.

The agreement is very good over many orders of magnitude - note that both axes use logarithmic scales. Tune shifts from analysis of data from application of the original map are shown as blue circles in Fig. 2. If we take the points from applying the original map, apply the transformation given by Eq. (5), and then repeat the Fourier analysis, we obtain the points shown as red crosses in Fig. 2. There is a negligible difference between the horizontal tune shift with vertical amplitude in the original map and in the normalized map. After applying the normalizing transformation, one of the dominant effects of the coupling (the tune shift in one plane with respect to amplitude in the other plane) remains.

Finally, we take the points constructed by applying the turn-independent normalizing transformation to the points generated by the original map, and apply the turndependent transformation Eq. (7). The results for the tune shifts resulting from this final transformation are shown by red squares in Fig. 2. We see there is still a strong dependence of the tune on the vertical amplitude, but we make two observations: first, for small vertical amplitudes the 

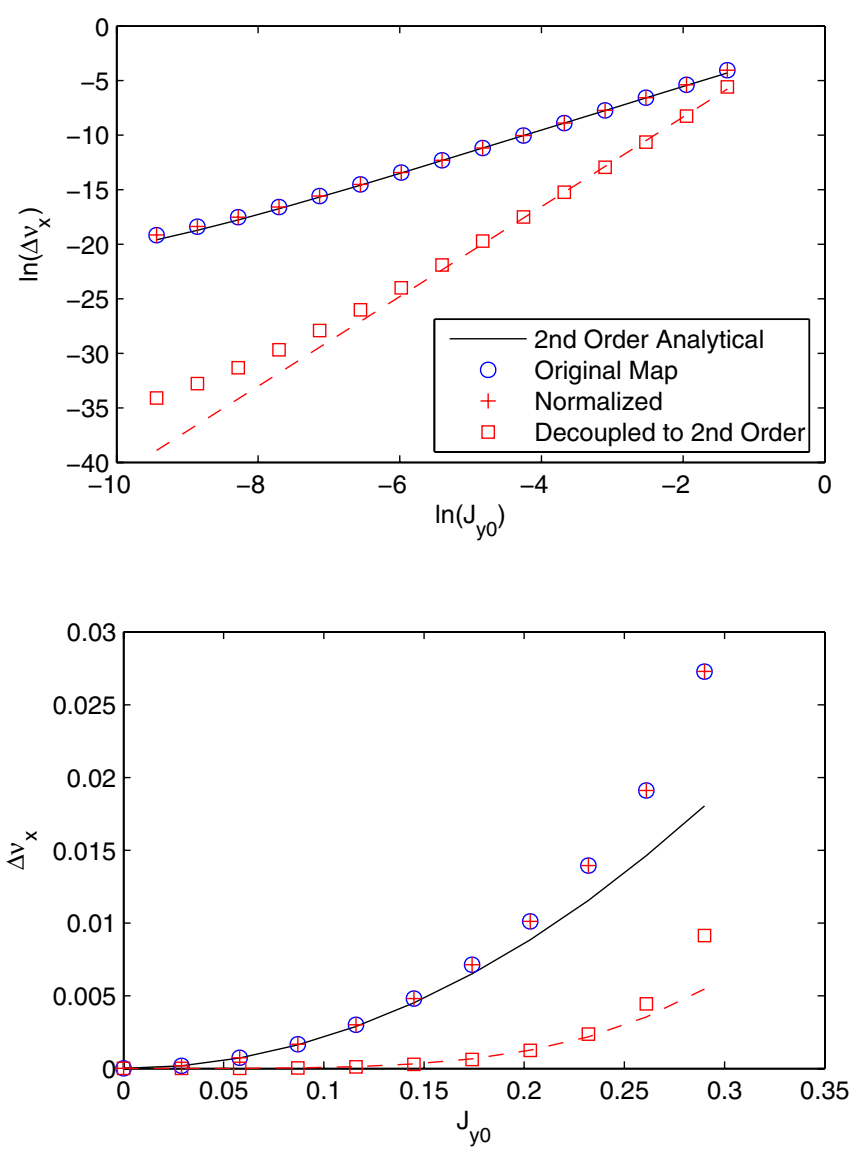

FIG. 2. (Color) Effect of nonlinear decoupling on horizontal tune shift with vertical amplitude. Top: logarithmic scales. Bottom: linear scales. The black line shows the analytical estimate of the tune shift from Eq. (8). The blue points show the result of a Fourier analysis of numerical mapping data, using the map of Eq. (7). Applying the turn-independent normalization Eq. (5) to the mapping data and then repeating the Fourier analysis gives the points shown by red crosses. Finally, applying the turn-dependent transformation Eq. (7) in addition to the turnindependent transformation gives the points shown as red squares. A fit to the final set of points (a straight line on the logarithmic plot) is shown as a broken red line.

tune shifts have been reduced by several orders of magnitude; and second, a line fitted to the points (the broken red line in Fig. 2) has gradient 4, indicating a fourth-order dependence of horizontal tune on vertical amplitude. Since we carried out the decoupling transformation only to third order in the generator of the map, these effects are exactly what we expect to find.

The difference between the black and broken red lines in Fig. 2 is an indication of the importance of higher-order terms in the map. For low vertical amplitudes, the dynamics are dominated by terms up to second order (in the action variables) in the generator. As the vertical amplitude is increased, the broken red line, indicating the contribution of the fourth-order terms to the dynamics, approaches the black line. Put another way, the difference between the lines indicates the rate of convergence of the normalizing transformation, expressed as a generator in power series form. Where the lines are well separated, the normalizing transformation converges quickly with a small number of terms in the generator; where the lines are close together, the normalizing transformation converges more slowly. One interesting observation in this respect is that in our investigations we found that the original map appears always to become unstable around the point where the solid black and broken red lines meet; for vertical amplitudes below this point, repeated applications of the map results in a set of points that remains bounded, whereas for vertical amplitudes above this point, repeated applications of the map results in divergence. Intuitively, this is entirely reasonable, since if the normalizing transformation does not converge, then the map is not well behaved, and stable dynamics cannot be expected.
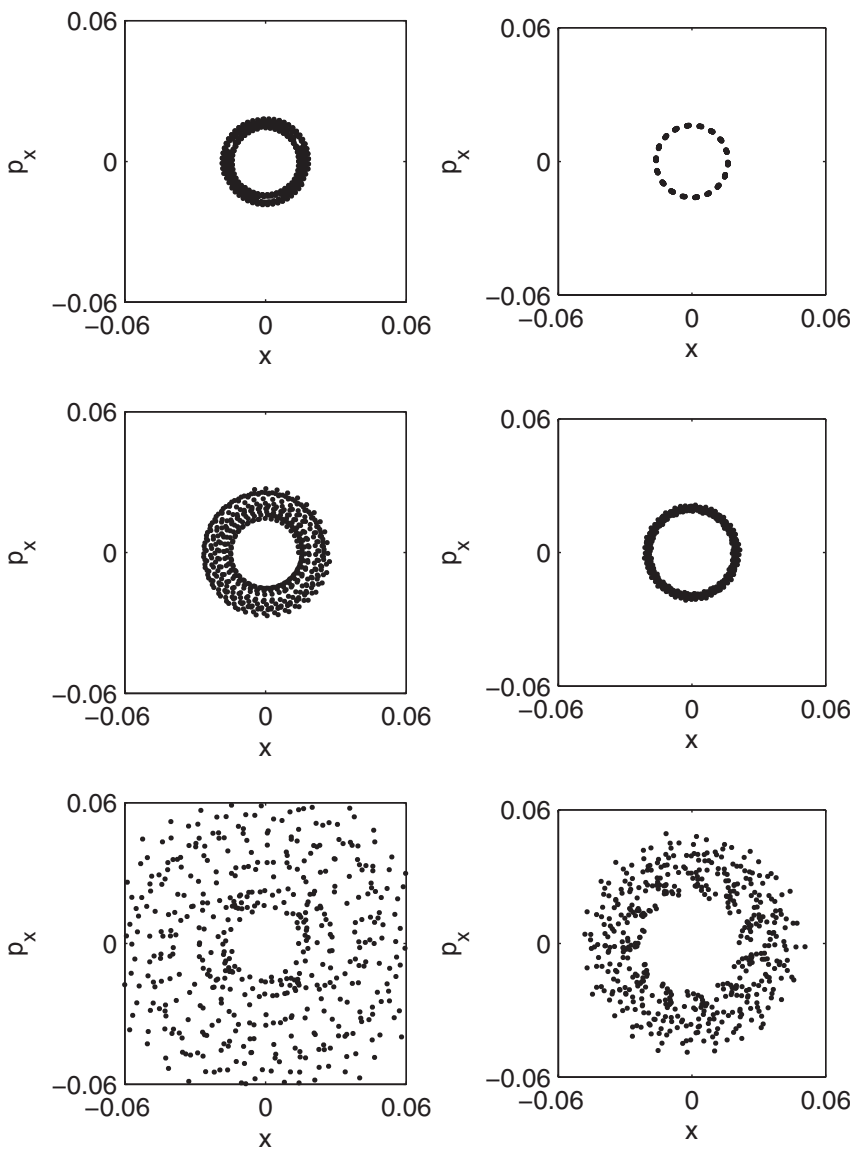

FIG. 3. Effect of normalizing and turn-dependent decoupling transformation on the horizontal phase space with different initial vertical amplitudes. The initial horizontal action is $10^{-4}$ in each case. The plots on the left show the phase space with the original map, Eq. (7), applied 1000 times. The plots on the right show the phase space with both the normalizing transformation, Eq. (5), and the turn-dependent decoupling transformation, Eq. (7), applied. Top: initial $J_{y}=0.08$. Middle: initial $J_{y}=$ 0.16. Bottom: initial $J_{y}=0.27$. 
To illustrate further the issue of convergence of the normalizing transformation, we can compare the phase space plots for points with different initial amplitudes. Such a comparison is shown in Fig. 3. As the vertical amplitude is increased, the residual scatter in phase space following a second-order normalization and third-order turn-dependent decoupling transformation also gets larger, showing the increasing significance of the higher-order terms. Close to the limit of dynamical stability, the residual scatter is very large because of the poor convergence of the normalizing transformation.

\section{CONCLUSIONS}

Our main purpose has been to discuss the features required of a transformation that will complete the decoupling of a map, in the sense of removing any dependence of the motion in one degree of freedom on the motion in another. In the case of a map with nonlinear coupling, we can use normal form analysis to construct a canonical transformation under which the motion appears as a set of regular ellipses in phase space; at this stage, we can identify the areas of these ellipses with conserved quantities. However, using time-independent (or turnindependent) transformations, there will in general remain terms in the map which couple motion between different degrees of freedom; specifically, the phase advance in one degree of freedom will depend on the amplitude of the motion in another degree of freedom.

Guided by the case of decoupling a Hamiltonian, we have shown that a turn-dependent canonical transformation (in effect, an infinite set of canonical transformations, indexed by the turn number) may be used to remove the remaining coupling terms in the generator of the map. There are indications in our numerical example that application of such a decoupling transformation can give some insight into the domain of stability of the original map.

Finally, we note that, as mentioned in the Introduction, use of turn-dependent canonical transformations allows us to construct variables in which Liouville's theorem is evident in each degree of freedom separately: when the system is completely decoupled, the area of phase space covered by a set of particles projected onto any degree of freedom is conserved, and Liouville's theorem, which requires the conservation of the phase space volume covered by the particles, is then clearly apparent.

\section{ACKNOWLEDGMENTS}

We thank Ina Reichel for translating Ref. [3] for us.

\section{APPENDIX A: LINEAR COUPLING}

\section{General Theory}

Consider a linear map given in the form of a Lie transformation [2]:

$$
M=\exp \left(: \mathbf{x}^{\mathrm{T}} \overline{\mathbf{m}} \mathbf{x}:\right)
$$

where $\overline{\mathbf{m}}$ is a symmetric matrix (we use a bar to indicate a matrix that appears in the generator of a Lie transformation), and the components of the vector $\mathbf{x}$ are the phase space variables. In $n$ degrees of freedom, $\overline{\mathbf{m}}$ is a $2 n \times 2 n$ matrix. The motion described by $M$ is coupled if $\overline{\mathbf{m}}$ has components outside the $2 \times 2$ block-diagonals: a decoupling transformation is one that removes the off blockdiagonal components of $\overline{\mathbf{m}}$.

A linear canonical transformation $F$ can be written in Lie operator form as

$$
F \cdot \mathbf{x}=\exp \left(: \mathbf{x}^{\mathrm{T}} \overline{\mathbf{f}} \mathbf{x}:\right) \cdot \mathbf{x}=\mathbf{f} \mathbf{x},
$$

where $\mathbf{f}$ is a matrix. Under $F$,

$$
M \mapsto \tilde{M},
$$

where

$$
\begin{aligned}
\tilde{M} & =F \cdot M \cdot F^{-1}=\exp \left(: F \cdot \mathbf{x}^{\mathrm{T}} \overline{\mathbf{m}} \mathbf{x}:\right) \\
& =\exp \left(: \mathbf{x}^{\mathrm{T}} \mathbf{f}^{\mathrm{T}} \overline{\mathbf{m}} \mathbf{f} \mathbf{x}:\right) .
\end{aligned}
$$

To decouple the motion, we need to find a matrix $\mathbf{f}$ that diagonalizes $\overline{\mathbf{m}}$. Such a matrix can be constructed from the eigenvectors of $\mathbf{S} \overline{\mathbf{m}}$, where $\mathbf{S}$ is the usual $2 n \times 2 n$ antisymmetric matrix with block diagonals,

$$
\mathbf{S}_{2}=\left(\begin{array}{cc}
0 & 1 \\
-1 & 0
\end{array}\right)
$$

Let $\mathbf{e}_{1}$ be the matrix of the eigenvectors of $\mathbf{S} \overline{\mathbf{m}}$, ordered and normalized so that

$$
\mathbf{e}_{1}^{\mathrm{T}} \mathbf{S e}_{1}=i \mathbf{S} .
$$

Then $\mathbf{f}$ can be written

$$
\mathbf{f}=\mathbf{e}_{1} \mathbf{T},
$$

where $\mathbf{T}$ is a $2 n \times 2 n$ block-diagonal matrix constructed from

$$
\mathbf{T}_{2}=\frac{1}{\sqrt{2}}\left(\begin{array}{cc}
i & 1 \\
i & -1
\end{array}\right)
$$

The generator $\overline{\mathbf{f}}$ of $F$ can be written

$$
\overline{\mathbf{f}}=\frac{1}{2} \mathbf{S e}_{2}\left(\ln \Lambda_{2}\right) \mathbf{e}_{2}^{-1},
$$

where $\mathbf{e}_{2}$ is the matrix of the eigenvectors of $\mathbf{f}$, and $\left(\ln \Lambda_{2}\right)$ is a diagonal matrix whose components are the logarithms of the eigenvalues of $\mathbf{f}$.

If the original map in matrix form is given by $\mathbf{m}$,

$$
M \cdot \mathbf{x}=\mathbf{m} \mathbf{x}
$$

then under the action of $F, \mathbf{m}$ transforms as

$$
\mathbf{m} \mapsto \tilde{\mathbf{m}}=\mathbf{f}^{-1} \mathbf{m f}
$$

and $\mathbf{f}^{-1} \mathbf{m f}$ is block diagonal: it is the decoupled map. 


\section{Numerical example}

As an example, consider the map in two degrees of freedom, given by

$$
\mathbf{m}=\mathbf{R}\left(\mu_{x}, \mu_{y}\right)\left(\begin{array}{cccc}
1 & 0 & 0 & 0 \\
0 & 1 & 0.2 & 0 \\
0 & 0 & 1 & 0 \\
0.2 & 0 & 0 & 1
\end{array}\right)
$$

where $\mathbf{R}\left(\mu_{x}, \mu_{y}\right)$ is a rotation matrix:

$$
\mathbf{R}\left(\mu_{x}, \mu_{y}\right)=\left(\begin{array}{cccc}
\cos \mu_{x} & \sin \mu_{x} & 0 & 0 \\
-\sin \mu_{x} & \cos \mu_{x} & 0 & 0 \\
0 & 0 & \cos \mu_{y} & \sin \mu_{y} \\
0 & 0 & -\sin \mu_{y} & \cos \mu_{y}
\end{array}\right) .
$$

$\mathbf{R}\left(\mu_{x}, \mu_{y}\right)$ represents a single-turn map for the transverse degrees of freedom in an uncoupled storage ring lattice with unit beta functions and phase advances $\mu_{x}, \mu_{y} ; \mathbf{m}$ represents the single-turn map when the storage ring is coupled by the introduction of a skew quadrupole into the lattice. We write

$$
\overline{\mathbf{m}}=\frac{1}{2} \mathbf{S e}_{0} \ln \left(\Lambda_{0}\right) \mathbf{e}_{0}^{-1},
$$

where $\mathbf{e}_{0}$ is a matrix constructed from the eigenvalues of $\mathbf{m}$ ordered and normalized so that

$$
\mathbf{e}_{0}^{\mathrm{T}} \mathbf{S e}_{0}=i \mathbf{S}
$$

and $\ln \left(\Lambda_{0}\right)$ is a diagonal matrix whose elements are the logarithms of the eigenvalues of $\mathbf{m}$. We then have that

$$
\mathbf{J}=\mathbf{m},
$$

where $\mathbf{J}$ is the Jacobian of the map $M$ :

$$
M=\exp \left(: \mathbf{x}^{\mathrm{T}} \overline{\mathbf{m}} \mathbf{x}:\right) .
$$

Let us take, in this example, $\mu_{x}=0.22 \times 2 \pi$ and $\mu_{y}=$ $0.385 \times 2 \pi$. Then, numerically,

$$
\mathbf{m} \approx\left(\begin{array}{cccc}
0.1874 & 0.9823 & 0.1965 & 0 \\
-0.9823 & 0.1874 & 0.0375 & 0 \\
0.1323 & 0 & -0.7501 & 0.6613 \\
-0.1500 & 0 & -0.6613 & -0.7501
\end{array}\right)
$$

and

$$
\overline{\mathbf{m}} \approx\left(\begin{array}{cccc}
-0.6998 & 0.0079 & 0.0133 & -0.1224 \\
0.0079 & -0.6968 & -0.0697 & 0.0791 \\
0.0133 & -0.0697 & -1.2059 & 0.0079 \\
-0.1224 & 0.0791 & 0.0079 & -1.2239
\end{array}\right) .
$$

Note that $\overline{\mathbf{m}}$ is symmetric, as expected, and is close to the diagonal matrix:

$$
\overline{\mathbf{r}}=-\frac{1}{2}\left(\begin{array}{llll}
\mu_{x} & & & \\
& \mu_{x} & & \\
& & \mu_{y} & \\
& & & \mu_{y}
\end{array}\right),
$$

where $\mathbf{x}^{\mathrm{T}} \overline{\mathbf{r}} \mathbf{x}$ is the generator of the map given in matrix form by $\mathbf{R}$. The difference between $\overline{\mathbf{m}}$ and $\overline{\mathbf{r}}$ is a result of the coupling.

Having obtained $\overline{\mathbf{m}}$, we can use the eigensystem of $\overline{\mathbf{m}}$ in Eq. (A1) to find

$$
\mathbf{f} \approx\left(\begin{array}{cccc}
0.9971 & 0 & -0.1043 & 0 \\
-0.0070 & 0.9957 & -0.1002 & -0.0694 \\
0.0698 & 0 & 1.0024 & 0 \\
-0.0997 & 0.1036 & 0.0104 & 0.9904
\end{array}\right) .
$$

Note that $\mathbf{f}$ is symplectic. Finally, we use the eigensystem of $\mathbf{f}$ in Eq. (A1) to find

$$
\overline{\mathbf{f}} \approx\left(\begin{array}{cccc}
-0.0035 & -0.0003 & -0.0500 & -0.0348 \\
-0.0003 & 0 & 0.0520 & 0 \\
-0.0500 & 0.0520 & 0.0052 & -0.0030 \\
-0.0348 & 0 & -0.0030 & 0
\end{array}\right) .
$$

Note that $\overline{\mathbf{f}}$ is symmetric. $\overline{\mathbf{f}}$ is also close to zero, which means that $\mathbf{f}$ is close to the identity; this is because, in this example, the coupling is small.

By direct matrix multiplication, we find

$$
\mathbf{f}^{\mathrm{T}} \overline{\mathbf{m}} \mathbf{f} \approx\left(\begin{array}{llll}
-0.6876 & & & \\
& -0.6876 & & \\
& & -1.2148 & -1.2148
\end{array}\right)
$$

and

$$
\begin{aligned}
\tilde{\mathbf{m}} & =\mathbf{f}^{-1} \mathbf{m} \mathbf{f} \\
& \approx\left(\begin{array}{cccc}
0.1943 & 0.9810 & 0 & 0 \\
-0.9810 & 0.1943 & 0 & 0 \\
0 & 0 & -0.7570 & 0.6534 \\
0 & 0 & -0.6534 & -0.7570
\end{array}\right) .
\end{aligned}
$$

Clearly, $\mathbf{f}$ decouples the map, as desired.

Figure 4 shows the results of "tracking" 1000 turns through the coupled storage ring lattice, represented by the map $\mathbf{m}$ given in Eq. (A2), starting with an arbitrary initial amplitude in $x$ and $y$; these points are shown in black. The coupling leads to a large scatter around an
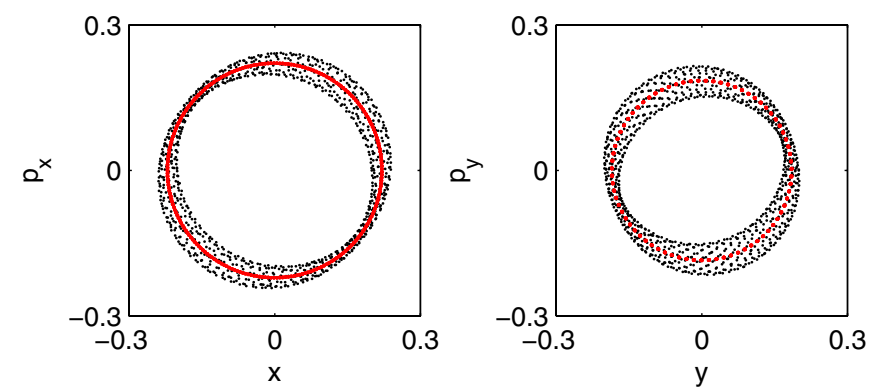

FIG. 4. (Color) Linear decoupling. Points showing the trajectory using the original coupled map are plotted in black. The same points, with a decoupling transformation applied, are plotted in red. 
"average" ellipse in each plane in phase space. To transform into the "uncoupled" coordinates, we apply the canonical transformation represented by $\mathbf{f}^{-1}$ thus

$$
\mathbf{x} \mapsto \tilde{\mathbf{x}}=\mathbf{f}^{-1} \mathbf{x} .
$$

The points thus obtained are plotted in red in Fig. 4. As expected, the decoupling transformation completely eliminates the scatter; the trajectory in each plane in phase space appears as an ellipse. There are conserved quantities clearly associated with geometric quantities, namely, the area of each red ellipse in phase space.

It appears that, given a coupled linear system, we are able to construct a canonical transformation that defines new variables in which the dynamics are uncoupled. However, the information on the coupling in the system (when described in the original variables) is not lost; it is simply the case that, rather than being contained in the map in the new variables, the information on the coupling is contained in the canonical transformation that we used to define the new variables. In other words, we describe the coupling not by means of a map, but by means of a canonical transformation that eliminates the coupling.

Finally, we note that the invariants of the coupled motion are readily obtained from the above process. In the de- coupled variables, the invariants are simply the areas of the ellipses (in this case, circles) in phase space, which can be written as $2 \pi J_{x}$ and $2 \pi J_{y}$, where

$$
\tilde{J}_{x}=\frac{1}{2}\left(\tilde{x}^{2}+\tilde{p}_{x}^{2}\right) \quad \tilde{J}_{y}=\frac{1}{2}\left(\tilde{y}^{2}+\tilde{p}_{y}^{2}\right) .
$$

Using Eq. (A3), we can substitute expressions in the original variables $\left(x, p_{x}, y, p_{y}\right)$ for the transformed variables $\left(\tilde{x}, \tilde{p}_{x}, \tilde{y}, \tilde{p}_{y}\right)$ in these equations, and immediately obtain quadratic invariants in the original variables.

[1] E. Forest, Beam Dynamics: A New Attitude and Framework (Harwood Academic Publishers, Chur, Switzerland, 1998), pp. 149-163.

[2] A. Dragt, Lie Maps, in Handbook of Accelerator Physics and Engineering, edited by A. W. Chao and M. Tigner (World Scientific, Singapore, 1999), pp. 76-82.

[3] H.D. Schräpel, Ingenieur Archiv (Archive Appl. Mech.) 48, 289 ( 1979), in German.

[4] H. Goldstein, Classical Mechanics (Addison-Wesley, Reading, MA, 1980), pp. 378-390. 